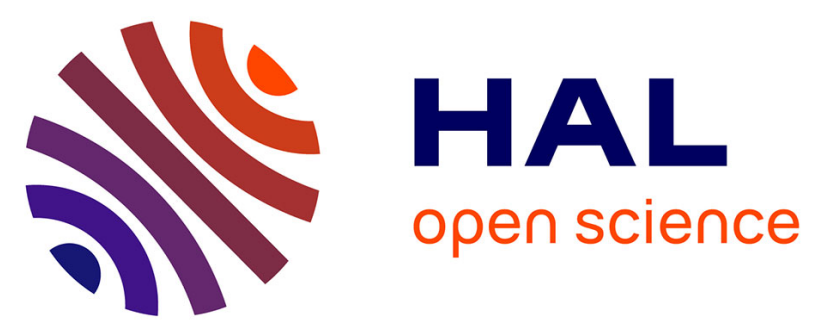

\title{
Wave patterns generated by an axisymmetric obstacle in a two-layer flow
}

Laurent Lacaze, Alexandre Paci, Emmanuel Cid, Sébastien Cazin, Olivier Eiff, J. G. Esler, E. R. Johnson

\section{- To cite this version:}

Laurent Lacaze, Alexandre Paci, Emmanuel Cid, Sébastien Cazin, Olivier Eiff, et al.. Wave patterns generated by an axisymmetric obstacle in a two-layer flow. Experiments in Fluids, 2013, vol. 54, pp. 1-10. 10.1007/s00348-013-1618-z . hal-00958121

\section{HAL Id: hal-00958121 \\ https://hal.science/hal-00958121}

Submitted on 11 Mar 2014

HAL is a multi-disciplinary open access archive for the deposit and dissemination of scientific research documents, whether they are published or not. The documents may come from teaching and research institutions in France or abroad, or from public or private research centers.
L'archive ouverte pluridisciplinaire HAL, est destinée au dépôt et à la diffusion de documents scientifiques de niveau recherche, publiés ou non, émanant des établissements d'enseignement et de recherche français ou étrangers, des laboratoires publics ou privés. 


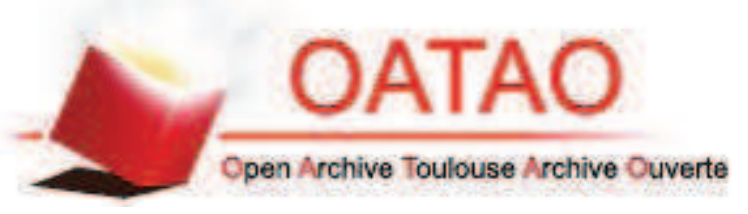

\section{Open Archive TOULOUSE Archive Ouverte (OATAO)}

OATAO is an open access repository that collects the work of Toulouse researchers and makes it freely available over the web where possible.

This is an author-deposited version published in : http://oatao.univ-toulouse.fr/ Eprints ID : 11161

To link to this article : DOI: $10.1007 / \mathrm{s} 00348-013-1618-\mathrm{Z}$

http://dx.doi.org/10.2516/ogst/2013183

To cite this version : Lacaze, Laurent and Paci, Alexandre and Cid, Emmanuel and Cazin, Sébastien and Eiff, Olivier and Esler, J. G. and Johnson, E. R. Wave patterns generated by an axisymmetric obstacle in a two-layer flow. (2013) Experiments in Fluids, vol. 54 $\left(\mathrm{n}^{\circ}\right.$ 1618). ISSN 0723-4864

Any correspondance concerning this service should be sent to the repository administrator: staff-oatao@listes-diff.inp-toulouse.fr 


\title{
Wave patterns generated by an axisymmetric obstacle in a two-layer flow
}

\author{
L. Lacaze $\cdot$ A. Paci $\cdot$ E. Cid $\cdot$ S. Cazin $\cdot$ \\ O. Eiff · J. G. Esler $\cdot$ E. R. Johnson
}

\begin{abstract}
Gravity waves generated by a moving obstacle in a two-layer stratified fluid are investigated. The experimental configuration is three-dimensional with an axisymmetric obstacle which is towed in one of the two layers. The experimental method used in the present study is based on a stereoscopic technique allowing the 3D reconstruction of the interface between the two layers. Investigation into the wave pattern as a function of the Froude number, $F r$, based on the relative density of the fluid layers and the velocity of the towed obstacle is presented. Specific attention is paid to the transcritical regime for which $F r$ is close to one. Potential energy trapped in the wave field patterns is also extracted from the experimental results and is analyzed as a function of both the Froude number, $F r$, and the transcritical similarity parameter $\Gamma$. In particular, a remarkable increase in the potential energy around $F r=1$ is observed and a scaling allowing to assemble data resulting from different experimental parameters is proposed.
\end{abstract}

L. Lacaze $(\bowtie) \cdot$ E. Cid · S. Cazin · O. Eiff

INPT, UPS, IMFT (Institut de Mécanique des Fluides de

Toulouse), Université de Toulouse, Allée Camille Soula,

31400 Toulouse, France

e-mail: laurent.lacaze@imft.fr

L. Lacaze $\cdot$ E. Cid $\cdot$ S. Cazin $\cdot$ O. Eiff

IMFT, CNRS, 31400 Toulouse, France

A. Paci

CNRM-GAME/GMEI (UMR3589 CNRS and Meteo-France),

42 av. G. Coriolis, 31057 Toulouse Cedex 1, France

e-mail: alexandre.paci@meteo.fr

J. G. Esler · E. R. Johnson

Department of Mathematics, University College London, Gower Street, London WC1E 6BT, UK

\section{Introduction}

Flow interaction with an immersed obstacle can not only lead to surface waves, but also to internal gravity waves if the fluid is density stratified. The case of an obstacle on the horizontal boundary of the flow is particularly interesting as it is a configuration similar to topographically generated internal gravity waves in geophysical flows. These waves are in particular studied for their role on mixing and global circulation in the ocean (Munk and Wunsch 1998; Tailleux 2009) and on momentum transport and drag in the atmosphere (Smith 1979; Wells and Vosper 2010). When the vertical stratification is such that internal gravity-wave energy is trapped and cannot propagate vertically, as at the bottom of the oceanic mixed layer (Apel et al. 1985; New and Pingree 1992) or at the top of the atmospheric boundary layer (Porter and Smyth 2002; Goler and Reeder 2004), waves can be studied as interfacial waves between two layers of fluid. Camassa and Tiron (2011) show that under certain condition, a two-layer model can be used to provide quantitative information on internal gravity waves propagating in a more general vertical stratification. Some recent laboratory experiments have focused on such waves in a two-layer flow, but they lack instantaneous 3D interface elevation measurements, the interface height being retrieved either from local probes (Johnson et al. 2006; Mercier et al. 2012) or from a vertical plane intersecting the interface (Knigge et al. 2010).

In the present study, an optical stereoscopic technique is deployed to measure an instantaneous field of the elevation of an interface located between two layer of fluid. Stereoscopic techniques have been widely used to measure surface elevation in free surface flows (Benetazzo 2006; Chatellier et al. 2010; de Vries et al. 2011; Douxchamps et al. 2005; Jehle et al. 2008). The technique is based on the 
determination of the position of different points, in the laboratory frame of reference, via their imaged positions in the image planes of two separate cameras. The threedimensional position of these points at different times thus yields quantitative information on the evolution of the free surface. However, tracking an interface is less straightforward than a free surface, because particles are spread vertically over the nonzero interface thickness. The main part of the algorithm used here was previously developed for the quantification of the sand bed evolution in the swash zone of a beach in a large-scale lab experiment (Astruc et al. 2012). In this previous study, dots aligned on a regular grid were projected on the beach. The center of the image dots on each camera plane were thus easily extracted and matched for 3D reconstruction. Here, the algorithm is extended to the case of random patterns using a crosscorrelation technique in order to measure an interface randomly seeded with particles. This method gives access, for the first time, to the full wave field induced by an obstacle towed on top of the upper layer of two layer of comparable depth in a large-scale lab experiment (Fig. 1). These experiments were inspired by recent theoretical works (Johnson and Vilenski 2005; Johnson et al. 2006; Esler et al. 2007) predicting the wave structure in similar flows where dispersive effects dominate over dissipative effects, as in many geophysical situations. Under certain conditions, for example, when the flow speed is close to the gravity-wave speed, these results lead to different predictions than previous work in the non-dispersive limit [e.g. (Schar and Smith 1993; Jiang and Smith 2000)].

In Sect. 2, the experimental set-up is presented, in particular a detailed description of the measurement technique and the associated validation. Section 3 is devoted to the gravity wave analysis. Finally, a discussion on the obtained results is proposed is Sect. 4.2.

\section{Experimental procedure}

\subsection{The experimental set-up}

The experiments are performed in the large stratified water flume at the geophysical fluid mechanics laboratory of CNRM-GAME in Toulouse. The flume is used here as a 22-m-long-, 3-m-wide- and 1-m-high- towing tank. The tank is filled with a stratified two-layer fluid made of saline water

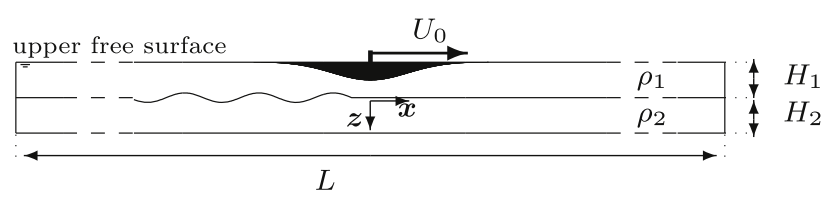

Fig. 1 Sketch of the experimental set-up $\left(L=22 \mathrm{~m}\right.$ and $H_{1} \approx H_{2} \approx$ $15 \mathrm{~cm}$ ) with two different concentrations of salt. The procedure to achieve the fluid stratification in the experimental tank is as follows. A two buckets system made of a salt water bucket and a fresh water bucket is used. The salt water bucket has a density equal to the desired lower layer density (numbered 2 in Fig. 1). The flume is filled up to a height $\mathrm{H}_{2}$ with fluid from the salt water bucket. In order to make the lighter upper layer (numbered 1 in Fig. 1), a pump system is used to mix salt water and fresh water from the two buckets with the desired volume fraction. This mixture is then diffused through floating diffusers on the free surface to make the top layer up to a height $H_{1}$. The diffuser procedure acts to sharpen the density profile at the interface. The two obtained saline layers are then characterized by their respective height $H_{i}$ and density $\rho_{i}, i=1$ corresponding to the layer in which the obstacle is immersed and $i=2$ the other layer (top and bottom layers in Fig. 1, respectively). Typical values are $H_{1} \approx 15 \mathrm{~cm}, \rho_{1}=1,000 \mathrm{~kg} \mathrm{~m}^{-3}, H_{2} \approx H_{1}$ or (slightly) larger and $\rho_{2}=1,059 \mathrm{~kg} \mathrm{~m}^{-3}$. As the above quantities evolve somewhat in time, they are systematically measured before each experiment. The total height $\mathrm{H}=\mathrm{H}_{1}+\mathrm{H}_{2}$ is fixed. All the experiments described in the following correspond to an interface thickness of no more than a few centimeters. A typical density profile $\rho(z)$, obtained with a conductivity probe, is shown in Fig. 2. In order to extract the vertical position and the thickness of the interface, the raw data are fitted with the function

$\rho=\frac{\rho_{2}-\rho_{1}}{2} \tanh \left(\frac{z-h_{i}^{f}}{e^{f}}\right)+\frac{\rho_{2}+\rho_{1}}{2}$,

where superscript $f$ denotes a fitting parameter and with $h_{i}$ and $e$ the vertical position and thickness of the interface,

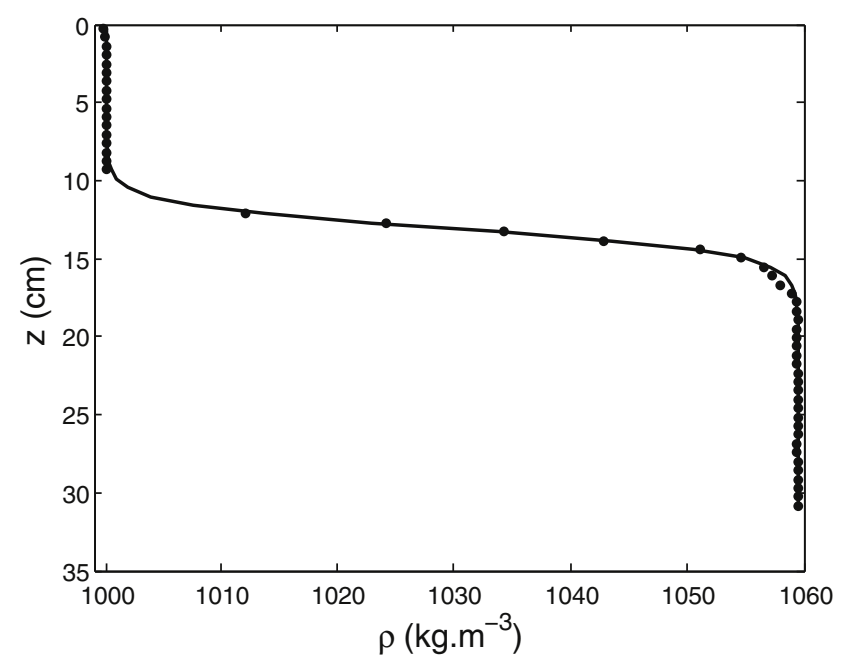

Fig. 2 Density variation $\rho$ along the vertical axis z. Dot symbols correspond to the experimental measurements and the solid line is the best fit estimated accordingly to Eq. (1) with $\rho_{1}=1,000 \mathrm{~kg} \mathrm{~m}^{-3}$, $\rho_{2}=1,059 \mathrm{~kg} \mathrm{~m}^{-3} \mathrm{~cm}$ and $h_{i}^{f}=12.84 \mathrm{~cm}$ 
respectively. Fitting parameters are then used to estimate the non-dimensional numbers of each experiments presented below. The density $\rho_{i}$ of each layer is obtained from direct measurements using an accurate densitometer. Superscripts $f$ are omitted in the following.

Two axisymmetric obstacles, referred to as obstacle $A$ and obstacle $B$ in the following, of base diameter $100 \mathrm{~cm}$ and maximum heights $h_{m}^{0}=7.7 \mathrm{~cm}$ and $h_{m}^{0}=12.5 \mathrm{~cm}$, respectively, are towed in the tank at velocity $U_{0}$ (see Fig. 1). The obstacles are made of cast resin. The obstacles are towed upside down in the top layer (see Fig. 1) to prevent wires and supports disturbing the interface. Therefore, the $z$ axis points downward and $z=0$ is set at the upper free surface. The $\boldsymbol{x}$ axis is aligned with the towing direction of the obstacle. In the following, the results are shown in the frame moving with the obstacle, $x=0$ being at the center of the obstacle. $y=0$ is set in the middle of the tank. In this reference frame, the radial profile of the obstacles is $h_{m}(r)=h_{m}^{0} \exp \left(-2 r^{2} / l^{2}\right)$ with $l=32 \mathrm{~cm}$ and $r$ the radial coordinate centered at $(x, y, z)=(0,0,0)$. It should be noted that $l=2 \sigma$, with $\sigma$ the standard deviation of the Gaussian function, corresponds to a characteristic horizontal length of the obstacle.

In this experimental configuration, three non-dimensional numbers are traditionally defined: the Froude number $F r=U_{0} / c$; the non-dimensional obstacle height ${ }^{1}$ $M=h_{m}^{0} / H_{1}$ and the aspect ratio $\delta=H_{1} / l$, where $c=\left(g^{\prime}\right.$ $\left.H_{1} H_{2} /\left(H_{1}+H_{2}\right)\right)^{1 / 2}$ is the phase velocity of the gravity wave evolving at the interface; $g^{\prime}=2 g\left(\rho_{2}-\rho_{1}\right) /$ $\left(\rho_{2}+\rho_{1}\right)$ is the reduced gravity. The phase speed $c$ is here defined for a two-layer fluid in the limit of zero interface thickness. This definition could be slightly affected by the interface thickness. However, as it will be seen in the following, this definition is accurate enough to characterize the wave pattern as a function of the $F r$ number and is therefore use for a sake of simplicity. A fourth parameter, $\Gamma=(F r-1) M^{-2 / 3}$, is known as the transcritical similarity parameter. The transcritical parameter $\Gamma$ was first introduce in the literature to describe the flow in the transcritical regime in which nonlinear effects always become important provided the flow is sufficiently close to critical. A measure of this distance to criticality therefore involves the blocking factor $M$ as well as the $F r$ number. It is shown in (Esler et al. 2007) that, in the transcritical regime, the flow is determined by the single similarity transcritical parameter for non-dispersive flow. The range of parameters of the different experiments performed here is given in Table 1 for obstacle $A$ and obstacle $B$.

\footnotetext{
${ }^{1}$ As obstacles are towed upside down, they cannot be fully immersed, so effective $h_{m}^{0}$ is slightly smaller than the values given above and is $7.5 \mathrm{~cm}$ (resp. $11.5 \mathrm{~cm}$ ) for obstacle $A$ (resp. obstacle $B$ ).
}

Table 1 Range of experimental parameters for obstacle $A$ (1st row) and obstacle $B$ (2nd row)

\begin{tabular}{lllll}
\hline & $F r$ & $M$ & $\delta$ & $\Gamma$ \\
\hline Obstacle $A$ & $0.24,1.44$ & $0.49,0.59$ & $0.48,0.40$ & $-1.22,0.63$ \\
Obstacle $B$ & $0.49,1.48$ & $0.90,1.60$ & $0.41,0.22$ & $-0.55,0.55$ \\
\hline
\end{tabular}

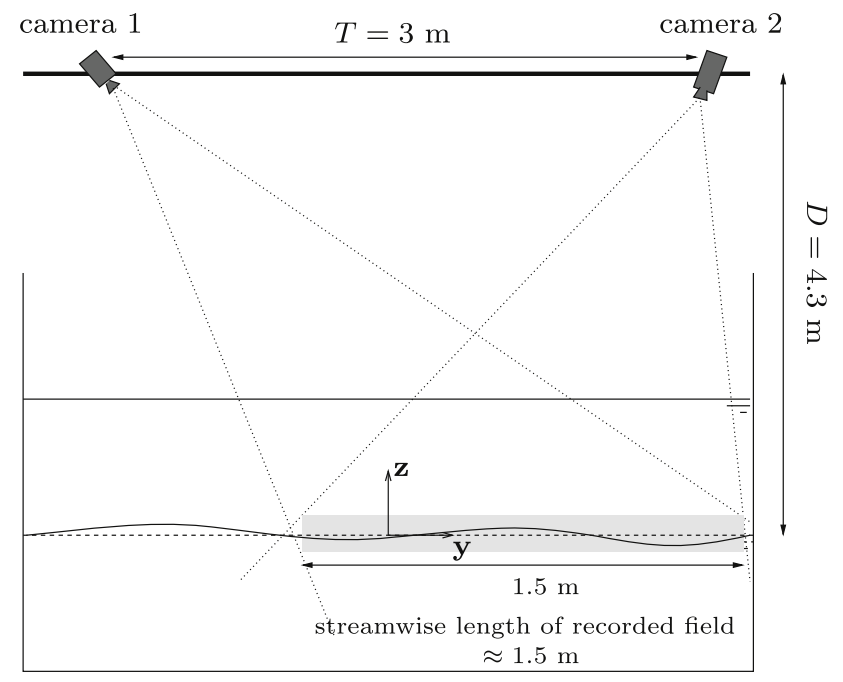

(frontal view)

Fig. 3 Sketch of the stereovision system set-up (not to scale) in the frontal $(y, z)$ plane. The two cameras image the interface of the twolayer fluid over $1.5 \times 1.5 \mathrm{~m}^{2}$. Dotted lines delineate the field of view of each camera. The horizontal dash line correspond to position of the interface at rest. The frontal projection of the measurement volume is represented by the gray area

In the present study, the stereo system was chosen to be fixed in the laboratory frame so that the whole wave field can be observed (giving access to $x \gg 1.5 \mathrm{~m}$ ). The measured zone is a $1.5 \times 1.5 \mathrm{~m}$ horizontal field. In the vertical direction, the deformation of the interface is of the order of few centimeters. Two synchronized high-resolution, highsensitivity 14-bit PCO-2000 cameras $\left(2,048 \times 2,048\right.$ pix $^{2}$ with a CCD cell size of $7.4 \times 7.4 \mu \mathrm{m}^{2}$ ) were chosen, coupled to $35 \mathrm{~mm}$ Nikon Nikkor lenses. The cameras were attached on a rigid and strong steel and aluminum structure, the distance between the cameras and from the zone of interest being about $T \sim 3 \mathrm{~m}$ and $D \sim 4.3 \mathrm{~m}$, respectively (see Fig. 3). The two cameras thus recorded the same field, at $2 \mathrm{~Hz}$, with an angle between the two line of sights of around $40^{\circ}$.

\subsection{The stereo system}

The stereoscopic method determines the position $P_{\mid w}=\left(x_{w}, y_{w}, z_{w}\right)$ in the world coordinate frame of a point $P$ in the laboratory frame by triangulation using images from two cameras, here referred to as cameras 1 and 2, 
fixed in the laboratory frame of reference and not aligned. The triangulation consists of identifying the point of intersection $P$ of the two rays passing through the image points $P^{1}$ and $P^{2}$ and the centers $O^{1}$ and $O^{2}$ of the optical systems associated with cameras 1 and 2 (see Fig. 4). This procedure thus retains the third spatial dimension, lost by imaging a scene with only one camera.

The stereo camera model and calibration process used here is similar to the one presented in Astruc et al. (2012) to which the reader is referred for details. The stereo procedure is organized in two main steps. First, a calibration is performed to obtain the transformation matrices linking the coupled pixel positions extracted from camera images and the $3 D$ spatial position. For such a large experimental field, the calibration is obtained by imaging a calibration plate of dimension $50 \times 50 \mathrm{~cm}^{2}$ in different parts of the scene. The calibration plate is made of one hundred white dots positioned on a regular grid. The accuracy of the distance between dots on the calibration plate is less than $100 \mu \mathrm{m}$. Position of the dot center is extracted from the images using image correlation method for shape recognition and a subpixel algorithm to improve the accuracy of the measurement. It was shown in Astruc et al. (2012) that 8 calibration image couples are sufficient to converge to the transformation function parameters with an accuracy of $<300 \mu \mathrm{m}$. The same procedure is therefore used here. Open source code of Bouguet (2004) is used to obtain the transformation matrices of the stereoscopic method. Once the calibration parameters are obtained, the second step consists in real measurements of the interface. Here, the interface is seeded with small polystyrene particles that can be detected on each camera image. Again, an image correlation method is used here as in Astruc et al. (2012). However, the specific experimental set-up of the stereo system used in the experiment and the stereo matched technique needed modification to extend the method to the case of a random seeded scene. In particular, a stereo-matching algorithm adapted to random pattern has been developed. As our scene exhibits different slopes, this implies that the images of the

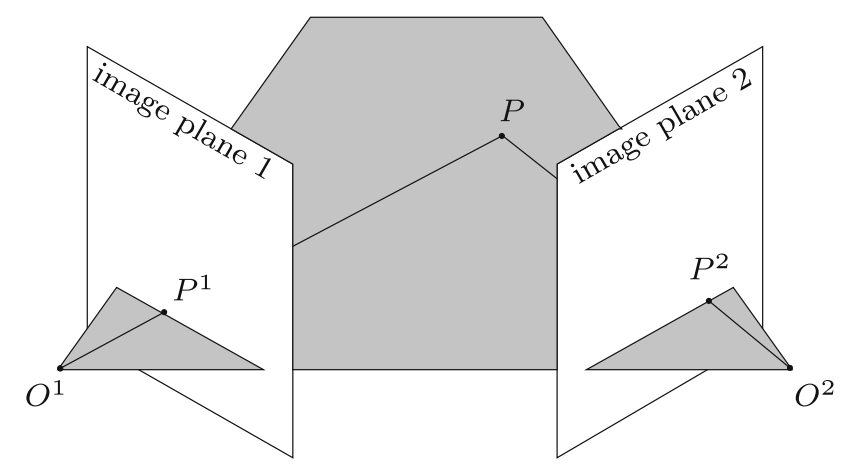

Fig. 4 Binocular stereovision configuration. The two image points $P^{1}$ and $P^{2}$ of the point $P$ are located on the line of sight of each camera same pattern acquired by the left and right cameras are often very different. This leads to failure in a matching process that uses simple cross-correlation operation between images from left end right cameras. In order to improve reliability our matching process is thus done in two steps. First, using the above calibration the homography matrix is calculated to transpose the left and right images in a common plane (Hartley and Zisserman 2004) using epipolar rectification. The mapping operation is thus reduced to a $1 \mathrm{D}$ search along the $\mathrm{x}$-axis on each image. We then applied a growing correspondence seeds algorithm (Cech and Sara 2007), using the previous mapping as a predictor to allow us to locally deform raw images in order to increase their similarity. The matching is then refined by cross-correlation with interrogation window size of $32 \times 32 \mathrm{pix}^{2}$. The cross-correlation, with image deformation, is applied twice using 'cubic spline' image interpolation scheme. Finally, the 3D reconstruction is adjusted to take into account the change of refractive index through the upper free surface crossed by the lines of sight to both cameras.

A validation of the method is performed using obstacle $A$ covered with random white dot patterns, which is placed upright on the bottom of a water layer of thickness $H=30 \mathrm{~cm}$, to take into account the presence of the free surface. The 3D reconstruction of the surface $h_{m}$ is shown in Fig. 5a. The accuracy of the method can then be estimated by comparing the averaged surface profile $<h_{m}>(r)$ with the theoretical profile $h_{m}^{0} \exp \left(-2 r^{2} / l^{2}\right), r$ being the radial coordinate centered at the summit of the obstacle and $\langle\cdot\rangle$ denoting the azimuthal averaging. The result is shown in Fig. 5b in which dots correspond to the mean profile $<h_{m}>(r)$. The error of the measured profile is estimated as being twice the standard deviation along the azimuthal direction and is shown in Fig. 5b. The obtained experimental profile is fitted with a function of form $h_{e} \exp \left(-2 r^{2} / l_{e}^{2}\right)$ with $h_{e}=7.716 \mathrm{~cm}$ and $l_{e}=31.65 \mathrm{~cm}$ (gray line in Fig. $5 \mathrm{~b}$ ). The error given by the two fit parameters is therefore of the order of $500 \mu \mathrm{m}$ while the error associated with the standard deviation gives a maximum error of $3 \mathrm{~mm}$ at the maximum slope of the obstacle. It has to be noted that the latter error slightly overestimates the error induced by the stereo method since it includes the accuracy of the manufactured shape, of the order of $1 \mathrm{~mm}$. The accuracy of the method is therefore well below the measured interface displacement which is of the order of few centimeters as shown below.

\section{Gravity wave pattern analysis}

\subsection{The stationary wave field}

Figure $6 \mathrm{a}-\mathrm{d}$ show the interface height deformation $\eta$ in the laboratory frame of reference $(X, Y)$ obtained at four 
(a)
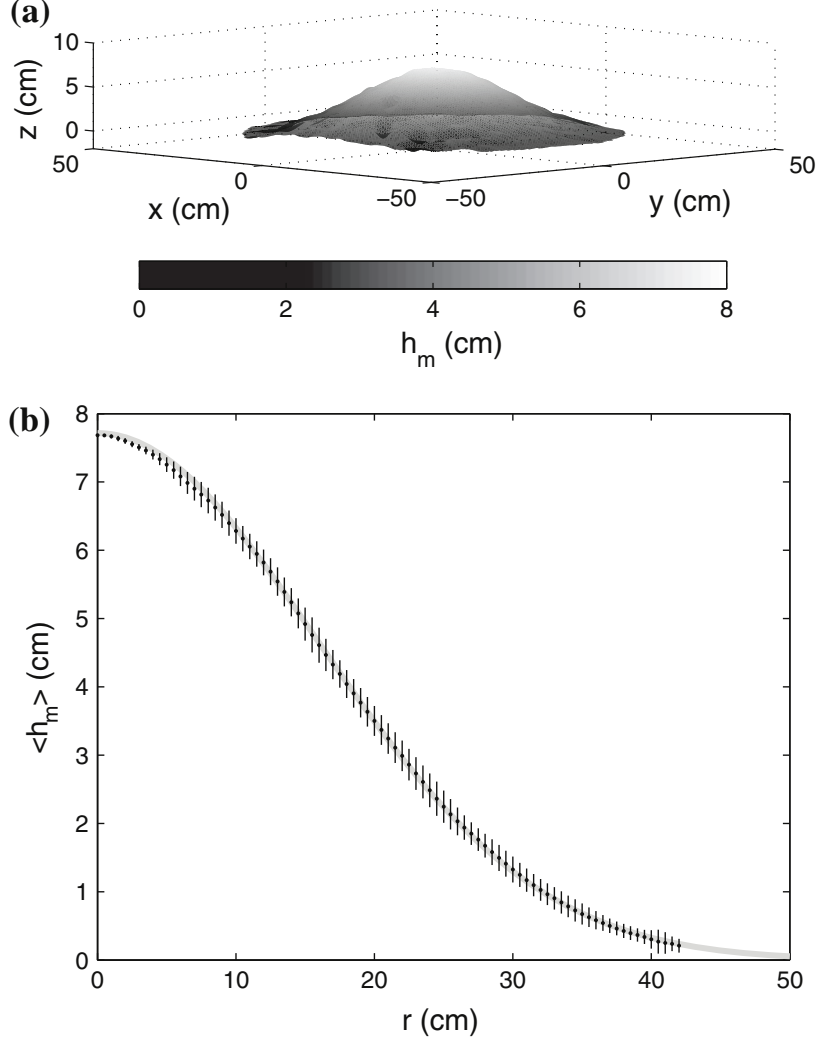

Fig. 5 3D reconstruction of the obstacle shape using the optical stereoscopic method: a contour of the obstacle in the world coordinate system, b averaged radial profile $\left\langle h_{m}\right\rangle$ (dots) and the associated standard deviation (vertical solid lines). (Gray solid line corresponds to the best fit as explained in the text.)

different times $t$, with an interval of 10 frames, i.e., $5 \mathrm{~s}$, between images (a), (b), (c) and (d) ${ }^{2}$. The next step of the experimental data analysis is the reconstruction of the interface height field in the frame of reference of the moving obstacle $(x, y)$. As the velocity $U_{0}$ of the towed obstacle is constant and waves are steady in the obstacle frame, the reconstruction consists only of shifting each measured field in the horizontal direction of propagation, i.e., $x=X-U_{0} t$, without any phase alignment between the different $\eta(X, Y, t)$ fields. To this end, the sampling rate ( $2 \mathrm{~Hz}$ ) has been chosen in order to ensure a spatial overlap of successive images in the obstacle frame of reference. The reconstruction resulting from Fig. $6 \mathrm{a}-\mathrm{d}$ is shown in Fig. 6e. A stationary wake (in the frame moving with the obstacle) is therefore obtained. There is some wave reflection from the side walls of the flume $(y=150 \mathrm{~cm})$ but this does not influence quantitative information extracted from the data. The latter point is discussed in the final section.

\footnotetext{
${ }^{2} t=0$ is defined here for the frame in which the obstacle center coincides with the center of the field of view.
}
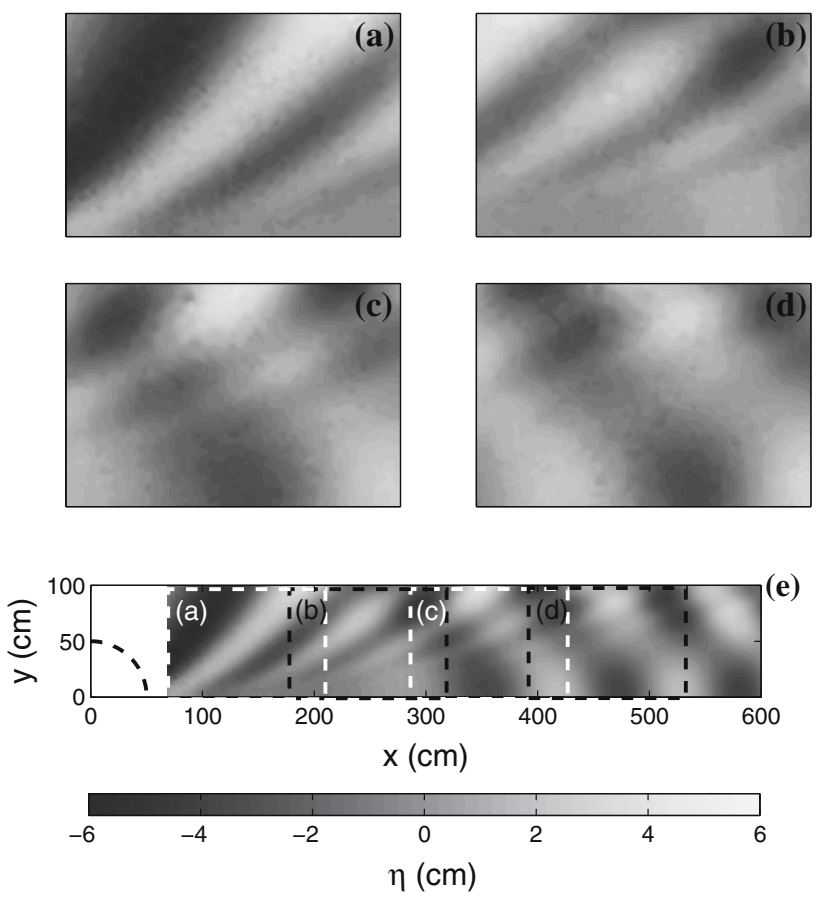

Fig. 6 Reconstruction of the stationary wave pattern in the frame of the obstacle for $F r=0.98, M=0.87(\Gamma=-0.02)$

\subsection{Validation and limitation of the experimental procedure}

Close to criticality for $F r \sim 1$, the flow undergoes recirculations and mixing close to the obstacle peak. This part of the wake has been observed visually from the side of the tank but is not observed on the measured wave pattern because the wake below the obstacle is not seen by the cameras in our experimental configuration. Moreover, the present optical technique is not adapted to extract quantitative measurements in an area where mixing occurs at the interface. Complementary technique using vertical laser sheet would be needed for this purpose. However, as the interface thickness $e$ evolves between experiments, it is important to evaluate its influence on the gravity wave characteristics. To this end, two experiments corresponding to different values of $e$ are compared, the other parameters being similar between the two experiments (see Fig. 7). For these two specific experiments, the parameters are $F r=0.98, M \approx 0.95$ (0.92 and 0.96, respectively) and $e /$ $H_{1}=0.2,0.3$, respectively. As observed in Fig. 7a, b, the evolution of $e / H_{1}$ is not significant enough to induce a modification of the wave pattern.

We focus here on the transcritical regime for which dispersive effects are known to affect the shape of the wave pattern in the wake of the obstacle when dissipative effects are small enough. Dispersion can be observed in the form of multiple solitary waves for instance (Johnson et al. 2006; 
(a)

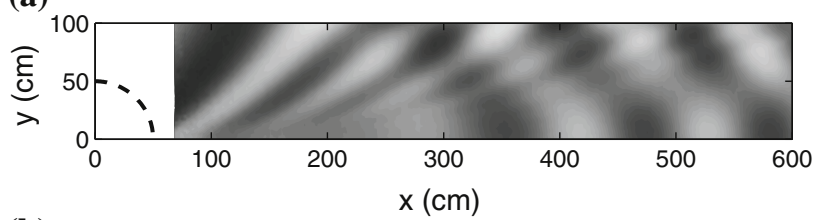

(b)

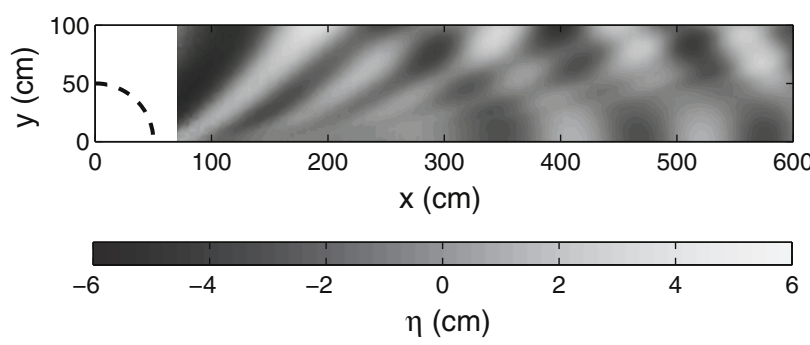

Fig. 7 Wave pattern for $F r=0.98, M \approx 0.95$ ( $\Gamma=-0.02)$; $e / H_{1}=0.2(\mathbf{a})$ and $e / H_{1}=0.3(\mathbf{b})$

Li et al. 2004; Maxworthy et al. 1984). The top layer depth to obstacle width ratio $\delta$ is a measure of this dispersion due to non-hydrostatic effects. We choose here a value of the parameter $\delta$ of about 0.4 to get moderate dispersive effects which do not override nonlinear effects but at the same time are dominant over viscous effects. Our purpose is to study this particular regime so $\delta$ is near constant in our experiments. Its influence on the following analysis is therefore considered to be negligible.

\subsection{Wake analysis}

Typical examples of gravity wave patterns obtained for different experimental parameters are shown in Fig. 8. Wave patterns generated by obstacle $A$ (resp. obstacle $B$ ) are shown in the left-hand side column (resp. right-hand side column). It has been chosen here to show the wave patterns in the $(M, \Gamma)$ parameter space. Therefore, each column corresponds to a given value of $M: M=0.6$ for obstacle $A$ and $M=0.9$ for obstacle $B$. Moreover, for each obstacle, the amplitude range of $\eta$ displayed is fixed in order to compare the relative wave deformation generated for different parameters. Each row in Fig. 8 corresponds to a constant value of the similarity transcritical parameter $\Gamma(\Gamma=[-0.55,-0.08,0.1]$ from top to bottom $)$. Nevertheless, the influence of the $F r$ on the wave pattern is clearly highlighted since it largely influences the value of $\Gamma$. In particular, a modification of the wave structures is observed from case to case in Fig. 8, first depending on the value of the $F r$ number. The evolution of the wave patterns in the wake of the obstacle as the $F r$ number increases, from Fig. 8a, b with $F r<0.7$ to Fig. 8e, f where $F r>1$, is shown. In particular, a transition to the $\mathrm{V}$-shock wake observed in the one shallow layer configuration at large $\mathrm{Fr}$ is observed (Jiang and Smith 2000).
The V-shaped wake appearing behind a moving object at the free surface of a liquid has been studied for a long time (Lighthill 1978). In particular, the ship wake angle is constant, known as Kelvin wake, when dispersive waves propagate at the free surface, i.e., in a deep ocean, while this angle decreases with the $F r$ number in the non-dispersive case, known as the Mach angle in a shallow layer configuration. More recently, in the case of boats evolving on the free surface of calm water, Rabaud and Moisy (2013) have highlighted the transition from a Kelvin wake to an equivalent Mach cone when the Froude number based on the horizontal length scale of the ship increases. Though, a deep ocean with dispersive waves is considered in their case. This behavior can be attributed to the cut-off wave length imposed by the horizontal ship size. In any case, the transition between the two regimes can be associated with a finite length scale in the system, horizontal or vertical. In a configuration similar to the present one, theoretical studies have highlighted the transition between these two wake angles as a function of a Froude number based on the phase velocity of the propagating waves. From our knowledge, no experimental evidence of this transition has been observed in a large facility allowing high Reynolds number regimes. The half wake angle $\alpha$ extracted from our experiments is reported as a function of $F r$ in Fig. 9. Two different behaviors can clearly be observed in this figure depending on the $F r$ number. For small $F r$, a constant angle which is typical of a Kelvin wake while for large $F r$, a Mach angle decreasing with $F r$. The qualitative wake evolution as a function of $F r$ is in accordance with the previous theoretical works previously mentioned (Hudimac 1961; Crapper 1967; Yeung and Nguyen 1999) and can therefore be associated with the dispersive nature of the forced waves which form the wake. At the small Fr, the dashed line shows the constant Kelvin angle while at large $F r$, dashed lines shows a $\sin ^{-1}\left(F r_{c} / F r\right)$ where $F r_{c}$ is a critical Froude number which is an adjustable parameter here. The two dashed lines correspond here to $F r_{c}=0.75$ and $F r_{c}=0.8$ which is slightly smaller than 1 . The critical Froude number $F r_{c}$ is less than 1 due to absence from the excited wave spectrum of waves significantly longer than the obstacle. These, being the fastest waves, would form the leading edge of the wake and in their absence the wake is narrower (Rabaud and Moisy 2013).

\subsection{Potential energy}

The energy that is injected in the wave field is quantified by the potential energy associated with the interface displacement. In the following, one defines the dimensional potential energy as 


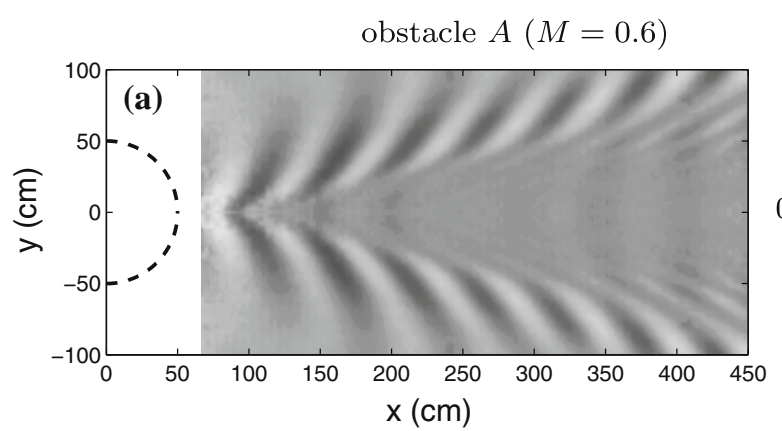

Fr

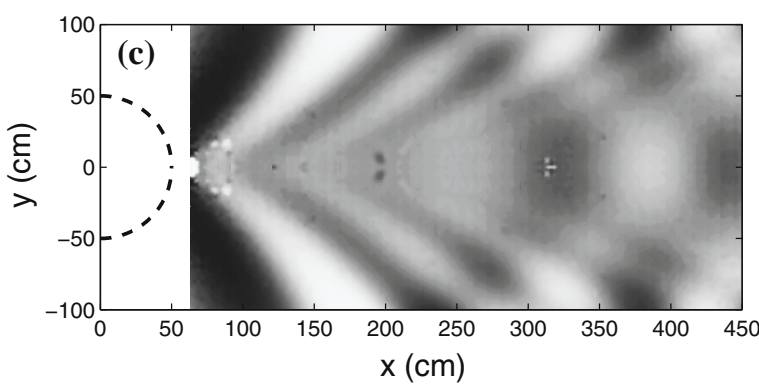

0.94

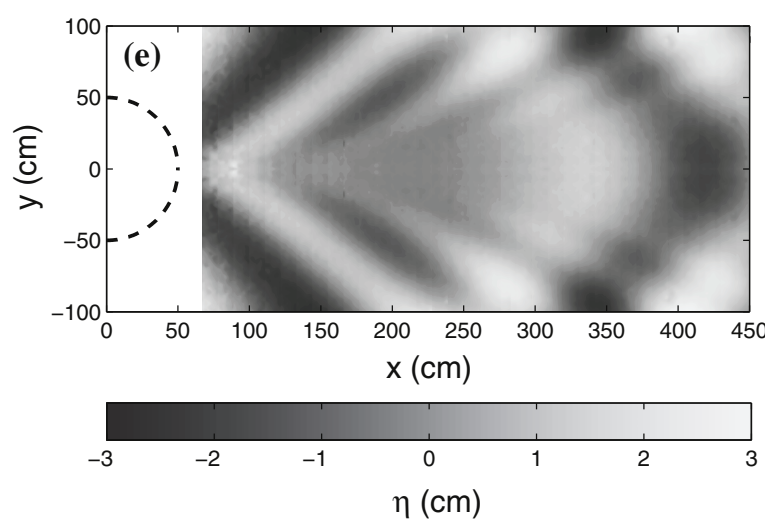

Fig. 8 Different gravity wave patterns for obstacle $A$ (left column), $M=0.6$, and obstacle $B$ (right-hand side column), $M=0.9 ;$ a $F r=0.62, \mathbf{b} F r=0.49, \mathbf{c} F r=0.94, \mathbf{d} F r=0.92$, e $F r=1.07$ and $\mathbf{f}$

$E p=\max _{\mathcal{D}\left(x_{m}\right)} \frac{1}{\left|\mathcal{D}\left(x_{m}\right)\right|} \int_{\mathcal{D}\left(x_{m}\right)} \frac{1}{2}\left(\rho_{2}-\rho_{1}\right) g \eta^{2} \mathrm{~d} S$

where $\left|\mathcal{D}\left(x_{m}\right)\right|$ is the horizontal area of region defined $\mathcal{D}\left(x_{m}\right)$ as $x<x_{m}$. A variable domain from case to case, $x_{m}$ being the streamwise position leading to a maximum of $E_{p}$, is preferred to a fixed domain since the significant spatial region of variation of the potential energy can depend on the regime considered, from subcritical to supercritical. ${ }^{3}$ In the following, different length scales are proposed to scale this potential energy. Two main length scales can be adopted. The first one is the layer depth $H_{1}$ of the fluid layer in which the obstacle is towed. We then define the

\footnotetext{
${ }^{3}$ It is verified that the obtained value of $x_{m}$ is always smaller than the distance at which reflective waves invade the domain.
}

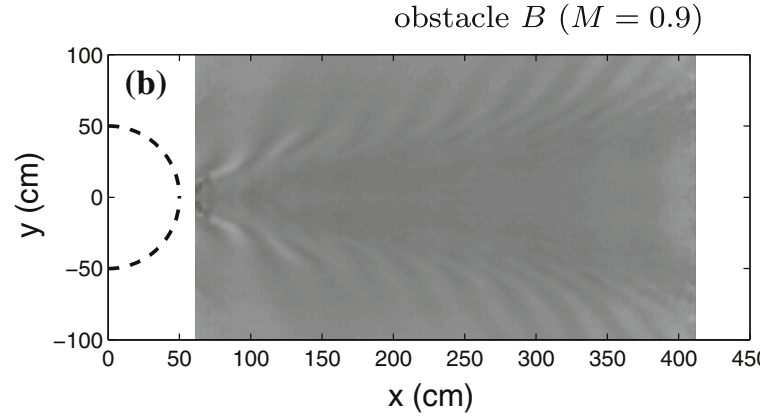

$F r$

0.49

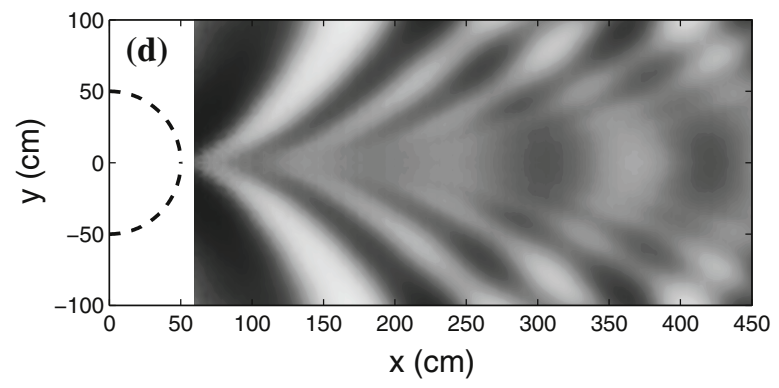

0.92

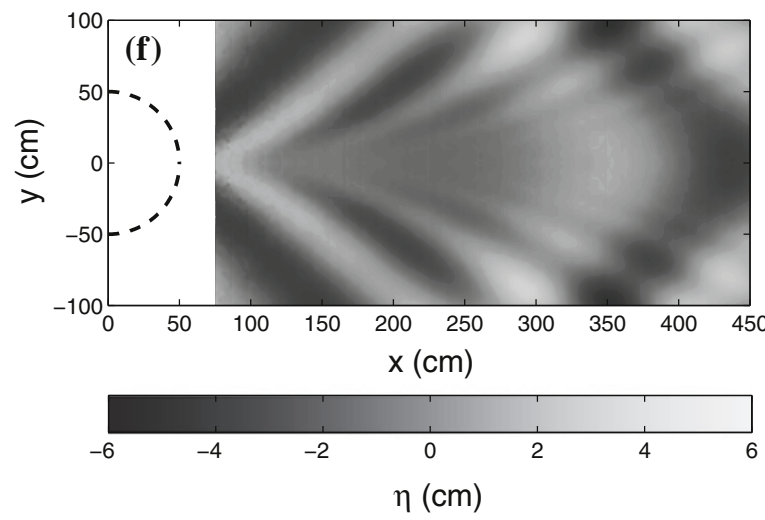

$F r=1.1$ (each row corresponds to a constant value of the similarity transcritical parameter $\Gamma$, with $\Gamma=[-0.55,-0.08,0.1]$ from top to bottom)

non-dimensional potential energy $E_{\mathrm{p}}^{(L H)}$ such as $E_{\mathrm{p}}^{(L H)}=$ $E_{\mathrm{p}} / \frac{1}{2}\left(\rho_{2}-\rho_{1}\right) g H_{1}{ }^{2}$. The second length scale is associated with the obstacle height $h_{m}^{0}$ leading to $E_{\mathrm{p}}^{(O H)}=E_{\mathrm{p}} / \frac{1}{2}\left(\rho_{2}-\right.$ $\left.\rho_{1}\right) g\left(h_{m}^{0}\right)^{2}$. These two non-dimensional potential energy are reported as a function of $F r$ in Fig. 10. Here, black dots show result for the smallest obstacle $A$ while gray symbols correspond to obstacle $B$. Gray symbols are subdivided into dark gray and light gray symbols corresponding to $M<1$ and $M \geq 1$, respectively. Peaks of potential energy around $F r=1$ are clearly highlighted. Note only the potential energy is presented here which is only part of the total energy. Figure 10 therefore highlights the increase in energy transferred to gravity waves around the critical regime $F r=1$, as it is found in the case of a single layer flow from the consideration of the wave drag (Esler et al. 2007; Wehausen 1973). Moreover, the range of $F r$ for 


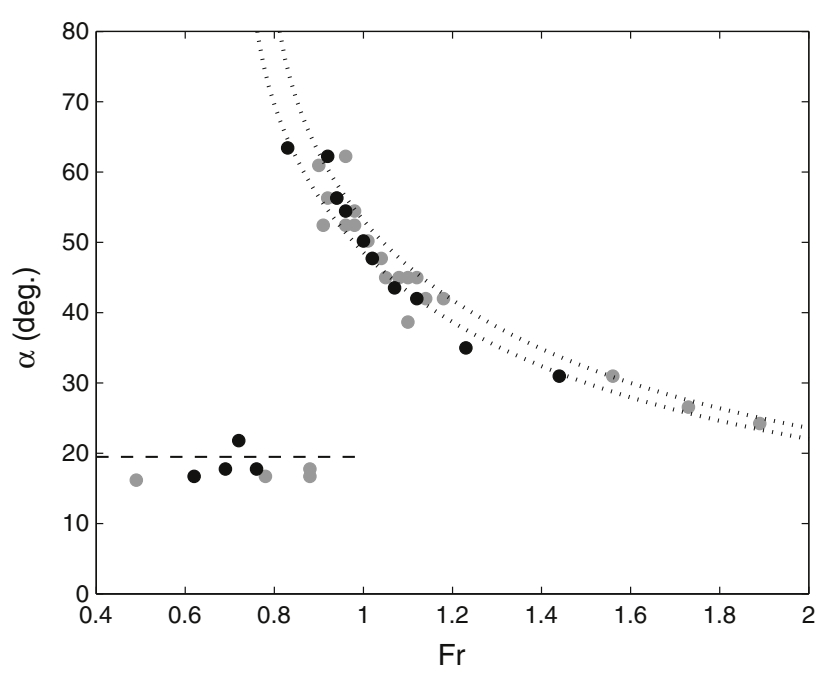

Fig. 9 Half wake angle $\alpha$ as a function of $F r$ for obstacle $A$ (black dots) and obstacle $B$ (gray dots). The dashed line correspond to the theoretical constant Kelvin angle of $19^{\circ} 5$. Dotted lines correspond to the Mach angle $\sin ^{-1}\left(F r_{c} / F r\right)$ where the adjustable parameter $F r_{c}$ is set here to 0.75 (lower curve) and 0.8 (upper curve)

which the value of the potential energy is significantly increased can be associated with the transcritical regime. This specific regime delimited by the subcritical regime and supercritical regime has been mostly studied in the case of single layer flows (Esler et al. 2007). In the set of experiments considered here, it is difficult to quantitatively delineate the different regimes. Nevertheless, wave patterns can be clearly distinguished for small $\mathrm{Fr}$ (typically $F r<0.7$ here) and large $F r$ (typically $F r>1.1$ here).

\section{Discussion}

\subsection{Similarity theory}

Based on previous theoretical results in which the influence of $\Gamma$ on the wake dynamics were highlighted (Esler et al. 2007), the experimental results can be characterized by the parameter $\Gamma$ instead of $\mathrm{Fr}$ and $\mathrm{M}$. For instance, wave patterns in Fig 8 are such that $\Gamma=[-0.55,-0.08,0.1]$ from top to bottom and independent of the considered obstacle (i.e., from one column to the other). Even if a difference in the relative normalized amplitude between $M=0.6$ and $M=0.9$ for $\Gamma=-0.55$ is observed, and probably attributed to the difference in $\mathrm{Fr}$ between these two experiments, the non-dimensional number $\Gamma$ is shown to be pertinent in characterizing the spatial structure of the wave patterns in the configuration studied here. Following this feature, a normalized potential energy is derived following the theoretical analysis of (Jiang and Smith 2000; Esler et al. 2007) in order to rescale the two set of experiments. In both
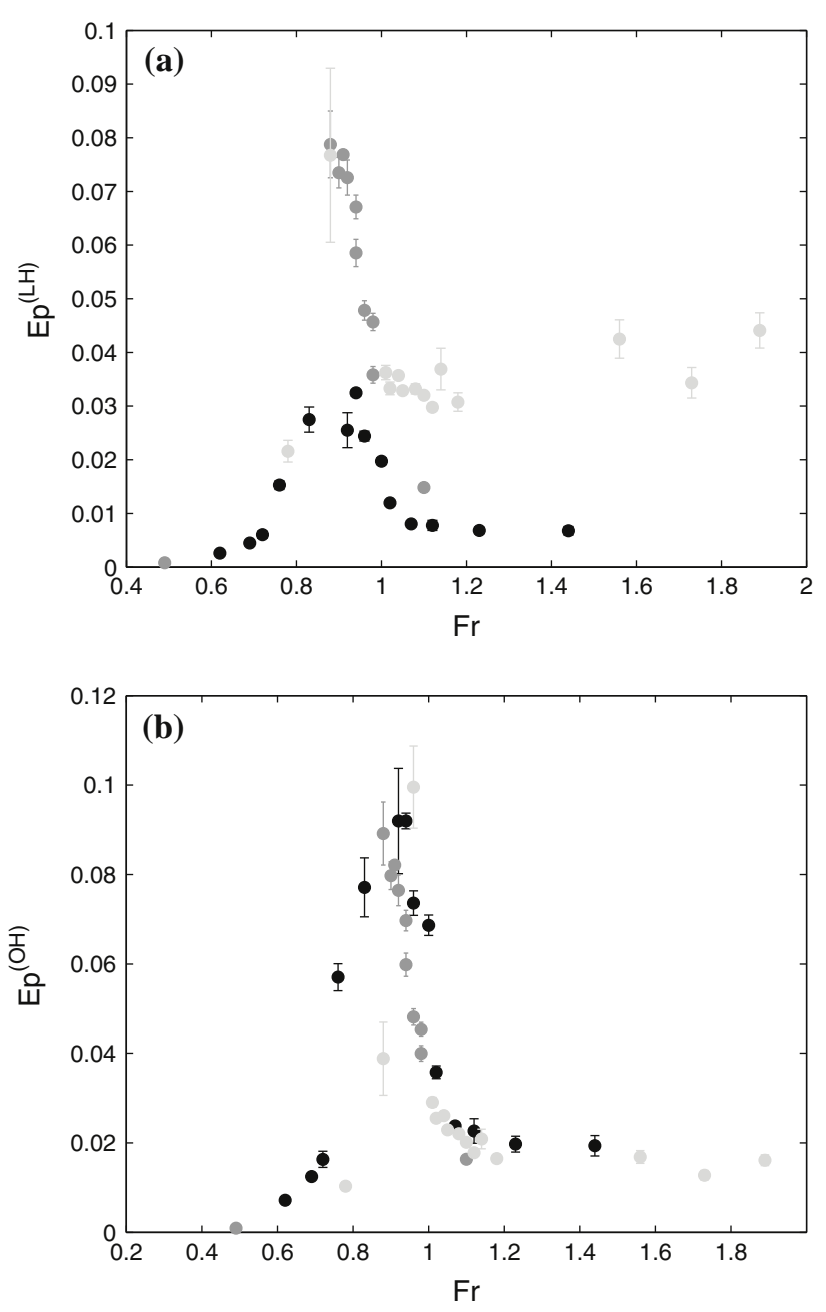

Fig. 10 Non-dimensional potential energy $E_{p}^{(L H)}(\mathbf{a})$ and $E_{p}^{(O H)}(\mathbf{b})$ as a function of $F r$ for obstacle $A$ (black dots) and obstacle $B$ (dark gray dots for $M<1$ and light gray dots for $M \geq 1$ )

cases, the case of a single layer is considered and the drag force exerted on the object is shown to scale as power law of $M$. In the first case (Jiang and Smith 2000), a linear analysis leads to a $M^{2}$ scaling while considering nonlinear effect leads to a $M^{5 / 3}$ scaling in the second case (Esler et al. 2007). Here, we adopt the same scaling for the potential energy. In particular, $E_{\mathrm{p}}{ }^{(O H)}$ and $E_{\mathrm{p}}{ }^{(L H)} / M^{5 / 3}$ are shown as a function of $\Gamma$ in Fig. 11. Note that $E_{\mathrm{p}}{ }^{(O H)}$ is consistent with the $M^{2}$ scaling since the obstacle height is used instead of the layer height to non-dimensionalized the potential energy. Surprisingly, these scalings seem to be pertinent for all $M$ considered in the present experiments while the theoretical analysis shows to be valid only for small $M$ (Esler et al. 2007). However, it does not seem obvious to validate the nonlinear theory more than the linear one with the data set available here. Nevertheless, Fig. 11 highlights the relevance of the normalization used for single layer flows and extended here to a two-layer flows. In particular, 

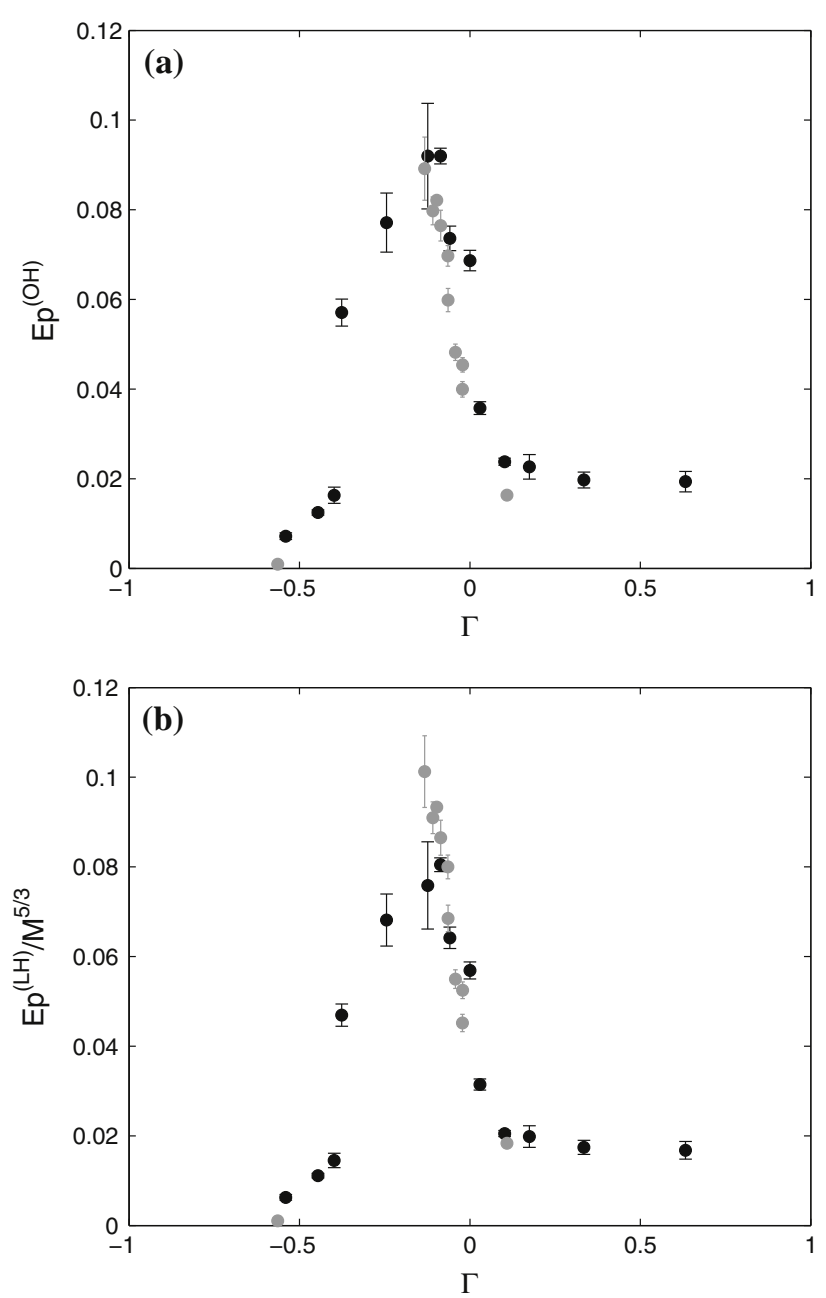

Fig. 11 Non-dimensional potential energy $E_{\mathrm{p}}{ }^{(O H)}\left(\right.$ a) and $E_{\mathrm{p}}{ }^{(L H)} / M^{5 / 3}$ (b) as a function of $\Gamma$ for obstacle $A$ (black dots) and obstacle $B$ (gray dots)

the different symbols define peaked curves whose maxima occur at the same value of $\Gamma \sim-0.15$.

\subsection{Reflected waves}

The experimental study presented in this paper shows the gravity wave patterns generated in the wake of an axisymmetric obstacle. Due to the geometry of the system considered here, waves are reflected on the side wall of the flume and invade the field of measurement at some distance downstream from the obstacle (as observed in Fig. 8). This behavior is more significant in the transcritical regime characterized by waves propagating nearly perpendicular to the streamwise direction. Even if these reflected waves do not affect the analysis proposed in this paper, a first attempt is proposed here to eliminate such reflective waves often observed in tanks experiments. To this purpose, a Hilbert transform filter is used here as explained in Mercier
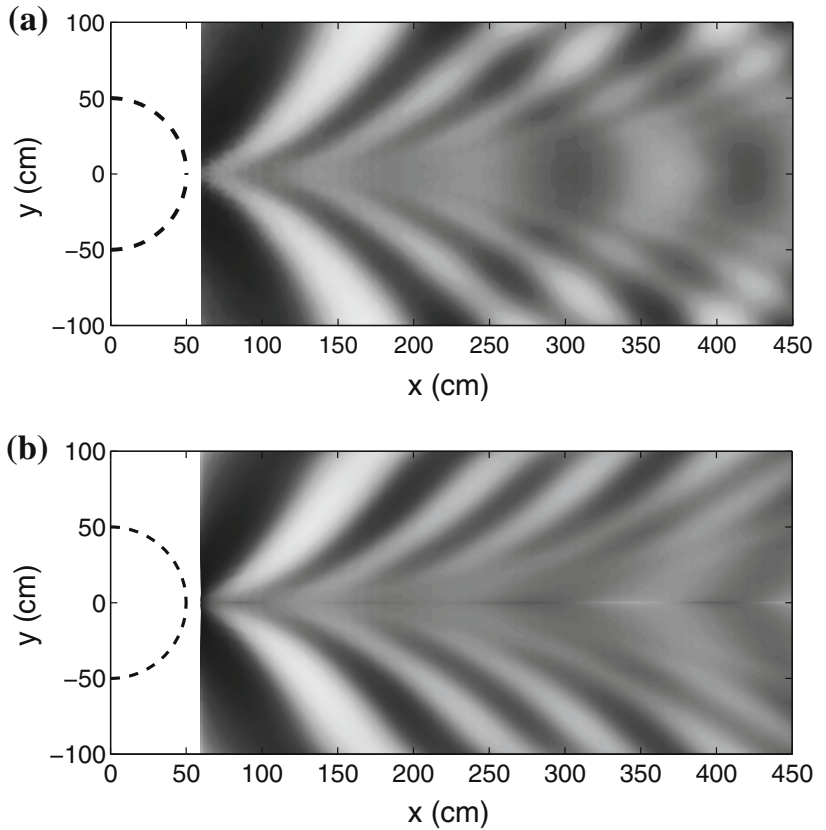

Fig. 12 Wave pattern for $\Gamma=-0.08, M \sim 0.9$ : raw data (a) and filtered data (b)

et al. (2008). Results are shown in Fig. 12 for the case $\Gamma=-0.08$ and $M=0.9$, i.e., in the transcritical regime [raw pattern (a) and filtered pattern (b)]. The spurious signal on the axis of symmetry $y=0$ is due to the small spatial extent of the measured field in the transverse direction $y$. However, the obtained filtered pattern is qualitatively convincing and appears to be pertinent in regions not too close to $y=0$. Note that the potential energy derived in the previous section was preferred to be calculated with the non-filtered results since the coordinate $x_{m}$ for which the potential energy (2) is maximum was always found to be smaller than the $x$ position at which reflective waves invades the domain of interest.

\section{Conclusion}

An optical stereoscopic technique has been deployed for the first time in large-scale laboratory experiments to measure an instantaneous field of surface elevation between two layers of miscible fluids. Two-dimensional gravity wave patterns forced by an obstacle in a two-layer fluid have been experimentally studied. In particular, attention has been paid to the influence of the Froude number $F r$ and the non-dimensional height $M$, or equivalently the transcritical similarity parameter $\Gamma$. General features associated with subcritical and supercritical fluid flows have been highlighted. It is moreover shown that close to the critical speed, a transcritical regime is characterized by a rapid evolution of the potential energy stored 
in the gravity waves. Moreover, in accordance to the case of a single layer, $M^{2}$ and $M^{5 / 3}$ drag scalings, obtained from linear and nonlinear analyses, respectively, are shown to be pertinent to merge the measured potential energy into a single curve as a function of the parameter $\Gamma$. Note that with the available data set, it is difficult to promote one scaling over the other. This stereo technique open new perspectives to study in the laboratory quasi-interfacial waves in stratified fluids.

Acknowledgments The experiments described in this publication were supported by the European Community's Sixth Framework Programme through the Grant to the budget of the Integrating Activity HYDRALAB III, Contract No. 022441(RII3). This document reflects only the authors' views and not those of the European Community. This work may rely on data from sources external to the HYDRALAB III project Consortium. Members of the Consortium do not accept liability for loss or damage suffered by any third party as a result of errors or inaccuracies in such data. The information in this document is provided "as is", and no guarantee or warranty is given that the information is fit for any particular purpose. The user thereof uses the information at its sole risk and neither the European Community nor any member of the HYDRALAB III Consortium is liable for any use that may be made of the information. We thank A. Belleudy, B. Beaudoin, B. Bourdelles, J.-C. Boulay, J.-C. Canonici, F. Murguet, M. Morera, S. Lassus Pigat and H. Schaffner of the CNRMGAME geophysical fluid mechanics laboratory in Toulouse for their kind support. We thank also Joseph Pearce and Orial Kryeziu for their participation in these experiments. The authors are grateful for helpful conversations with M. Mercier and F. Stoop.

\section{References}

Apel J, Holbrook J, Liu A, Tsai J (1985) The Sulu sea internal soliton experiment. J Phys Oceanogr 15(12):1625-1651

Astruc D, Cazin S, Cid E, Eiff O, Lacaze L, Robin P, Toublanc F, Cáceres I (2012) A stereoscopic method for rapid monitoring of the spatio-temporal evolution of the sand-bed elevation in the swash zone. Coast Eng 60:1-20

Benetazzo A (2006) Measurements of short water waves using stereo matched image sequences. Coast Eng 53:1013

Bouguet JY (2004) Camera calibration toolbox for Matlab. http:// www.vision.caltech.edu/bouguetj/calib_doc/

Cech J, Sara R (2007) Efficient sampling of disparity space for fast and accurate matching. In: Proceedings of BenCOS Workshop CVPR

Camassa R, Tiron R (2011) Optimal two-layer approximation for continuous density stratification. J Fluid Mech 669:32-54

Chatellier L, Jarny S, Gibouin F, David L (2010) Stereoscopic measurement of free surface flows. In: 14th international conference on experimental mechanics, Poitiers, France

Crapper GD (1967) Ship waves in a stratified ocean. J Fluid Mech 29:667

de Vries S, Hill DF, de Schipper MA, Stive MJF (2011) Remote sensing of surf zone waves using stereo imaging. Coast Eng 58:239

Douxchamps D, Devriendt D, Capart H, Craeye C, Macq B, Zech Y (2005) Stereoscopic and velocimetric reconstructions of the free surface topography of antidune flows. Exp Fluids 39:533

Esler JG, Rump OJ, Johnson ER (2007) Non-dispersive and weakly dispersive single-layer flow over an axisymmetric obstacle: the equivalent aerofoil formulation. J Fluid Mech 574:209
Goler RA, Reeder MJ (2004) The generation of the morning glory. J Atmos Sci 61:1360-1376

Hartley RI, Zisserman A (2004) Multiple view geometry in computer vision. Cambridge University Press, Cambridge

Hudimac AA (1961) Ship waves in a stratified ocean. J Fluid Mech $11: 229$

Jehle M, Jarny S, David L (2008) Différentes approches pour la mesure d'interfaces et de surface libre. In: 11ème Congrès Francophone de Techniques Lasers, Poitiers, France. p 225. (in French)

Jiang Q, Smith RB (2000) V-waves, bow shocks, and wakes in supercritical hydrostatic flow. J Fluid Mech 406:27

Johnson ER, Vilenski GG (2005) Two-dimensional leaps in nearcritical flow over isolated orography. Proc R Soc Lond A 61(2064):3747-3763

Johnson ER, Esler JG, Rump OJ, Sommeria J, Vilenski GG (2006) Orographically generated nonlinear waves in rotating and nonrotating two-layer flow . Proc R Soc Lond A 462(2065):3-20

Knigge C, Etling D, Paci A, Eiff O (2010) Laboratory experiments on mountain induced rotors. Q J R Meteorol Soc 136:442-450

Li X, Dong C, Clemente-Colón P, Pichel WG, Friedman KS (2004) Synthetic aperture radar observation of the sea surface imprints of upstream atmospheric solitons generated by flow impeded by an island. J Geophys Res 109. doi:10.1029/2003JC002168

Lighthill G (1978) Waves in fluids. Cambridge university press, Cambridge

Maxworthy T, Chabert d'Hieres H, Didelle H (1984) The generation and propagation of internal gravity waves in a rotating fluid. J Geophys Res 89: doi:10.1029/JC089iC04p06383

Mercier MJ, Garnier NB, Dauxois T (2008) Reflexion and diffraction of internal waves analyzed with the Hilbert transform. Phys Fluids 20:086601

Mercier MJ, Mathur M, Gostiaux L, Gerkema T, Magalhes JM, Da Silva JCB, Dauxois T (2012) Soliton generation by internal tidal beams impinging on a pycnocline: laboratory experiments. J Fluid Mech 704:37-60

Munk W, Wunsch C (1998) Abyssal recipes II : energetics of tidal and wind mixing. Deep Sea Res Part I Oceanogr Res Pap 45(12): 1977-2010

New A L, Pingree RD (1992) Local generation of internal soliton packets in the central Bay of Biscay. Deep Sea Res Part A Oceanogr Res Pap 39(9):1521-1534

Porter A, Smyth NF (2002) Modelling the morning glory of the Gulf of Carpentaria. J Fluid Mech 454:1-20

Rabaud M, Moisy F (2013) Ship wakes: Kelvin or Mach angle. Phys Rev Lett 110:214503

Smith RB (1979) The influence of mountains on the atmosphere. Adv Geophys 33:87-230

Tailleux R (2009) On the energetics of stratified turbulent mixing, irreversible thermodynamics, boussinesq models and the ocean heat engine controversy. J Fluid Mech 638:339-382

Schar C, Smith RB (1993) Shallow-water flow past an isolated topography. Part I: Vorticity production and wake formation. J Atmos Sci 50:1373-1400

Wehausen JV (1973) The wave resistance of ships. Adv Appl Mech 13:93-245

Wells H, Vosper SB (2010) The accuracy of linear theory for predicting mountain-wave drag: implications for parametrization schemes. Q J R Meteorol Soc 136:429-441

Yeung RW, Nguyen TC (1999) Waves generated by a moving source in a two-layer ocean of finite depth. J Eng Math 35:85 